\title{
Facteurs influant sur la validité de l'épreuve à l'alcool utilisée pour la sélection des laits stables à la chaleur
}

\author{
par \\ F. METRO, M. J. DESMAZEAUD, O. CERF \\ Laboratoire de Microbiologie Laitière et de Génie Alimentaire \\ Institut National de la Recherche Agronomique \\ F78350 Jouy-en-Josas (France)
}

\section{INTRODUCTION}

La stabilité du lait à l'alcool est traditionnellement utilisée comme critère de sélection des laits destinés à subir un traitement thermique. Ainsi il est courant de ne traiter à ultra-haute température que les laits stables lors de "l'épreuve à l'alcool à $75^{\circ}$ Gay-Lussac " (d'autres critères pouvant d'ailleurs être utilisés simultanément).

Toutefois cette habitude, qui s'appuie pourtant sur une longue expérience pratique, ne donne pas satisfaction en toutes circonstances. $\mathrm{Au}$ fur et à mesure que la proportion de lait pasteurisé diminue au profit de celle des laits de longue conservation, il apparaît de plus en plus que dans certaines zones géographiques, ou à certaines saisons, l'épreuve à l'alcool renseigne mal sur la stabilité réelle du lait à la chaleur.

La stabilité du lait à la chaleur a fait l'objet de nombreux travaux de recherches portant essentiellement sur des laits individuels, qui ont été passés en revue par Fox et Morrissey (1977). Dans beaucoup de ces travaux, les auteurs ont cherché une relation entre la stabilité à la chaleur et la composition du lait : une seule relation univoque a été démontrée avec la concentration en urée. On a également noté que la stabilité à la chaleur augmente lorsque le rapport du calcium soluble (ou du calcium ionisé) au phosphate soluble diminue. Aucun des autres facteurs étudiés n'est lié de façon simple, reproductible et incontestable à la stabilité à la chaleur.

La stabilité du lait à l'alcool n'a été que très peu étudiée à notre connaissance. On peut citer les résultats de Davies et White (1958), montrant que la stabilité à l'alcool diminue lorsque la concentration 
en calcium ionisé augmente. Ceci a été confirmé par Iizuka (1960) Batra et de Man (1964), Puri, Muraki et Toteja (1965), Labuschagne (1968) et Labuschagne et Landrey (1969). Par ailleurs Habaj et al. (1974) ont mis en évidence une anomalie de composition de la caséine K et de la $\beta$-lactoglobuline dans des laits individuels instables à l'alcool. Zhdanova et Alekseeva (1969) n'ont pas trouvé de relation nette entre la composition du lait et sa stabilité à l'alcool. Toit, Lück et Tonder (1970) et Jooste et Groeneveld (1971) après d'autres auteurs ont noté une bonne corrélation entre la stabilité à l'alcool du lait pré-incubé et le nombre initial de bactéries.

En ce qui concerne la stabilité à l'alcool comme critère de stabilité à la chaleur, encore moins d'études systématiques semblent avoir été faites. Alors que Kisza, Kruk et Roskosz (1974) et Claesson, Andersson et Claesson (1974) trouvent une corrélation entre les deux stabilités, Vaitkus et al. (1974) pour leur part concluent que la stabilité à l'alcool ne peut être un guide sûr pour connaître la stabilité à la chaleur. Quant à Belousov, Surotsev et Rossikhina (1971), ils observent que des laits stables à l'alcool peuvent être instables à la chaleur, et réciproquement.

Les recherches citées ci-dessus peuvent être rangées en deux catégories : celles qui montrent des relations entre composition et stabilité, celles qui tentent d'expliquer les mécanismes de la stabilité ou de l'instabilité. Seuls quelques travaux sur la stabilité à la chaleur appartiennent à la seconde catégorie (Fox et Morrissey, 1977). Par conséquent, s'il existe une causalité commune à la déstabilisation du lait par la chaleur et par l'alcool, elle n'a apparemment été ni recherchée ni prouvée. D'autre part les travaux sur la stabilité à l'alcool, comme une proportion importante de ceux sur la stabilité à la chaleur, sont anciens dans la mesure où ils ont été faits avec des laits ayant subi des traitements technologiques sans rapport avec ceux auxquels sont soumis actuellement les laits de grand mélange : réfrigération à la ferme, croissance d'une flore psychrotrophe, transferts mécaniques brutaux, long intervalle entre la traite et l'arrivée à l'usine, etc.

Nous avons donc été amenés à étudier si l'épreuve à l'alcool était ou non un bon indicateur de la stabilité du lait au chauffage. Le présent travail porte sur la stabilité du lait lorsque (celui-ci ayant été le siège d'une croissance de micro-organismes semblables à celle se produisant dans les laits mal réfrigérés et/ou conservés trop longtemps au froid) une modification du $\mathrm{pH}$ ou une protéolyse a eu lieu. Plutôt que de comparer des laits d'origines différentes, nous avons préféré utiliser un même lait dans lequel divers micro-organismes ont été cultivés. En outre, l'effet de modifications de la teneur du lait en matière sèche a également fait l'objet d'une expérimentation.

La question de la déstabilisation des caséines durant la conservation du lait après stérilisation n'a pas été examinée. 


\section{MATERIEL ET METHODES}

\section{Caractéristiques du lait utilisé}

\section{Origine du lait sec écrémé}

La poudre Nilac (Nizo, Pays-Bas) a été choisie pour la préparation du lait expérimental. Il s'agit d'une poudre fabriquée par un procédé "basse température " qui est garantie exempte d'antibiotiques. La quantité de matière grasse résiduelle est inférieure à 1 p. 100 .

\section{Reconstitution}

Le lait était reconstitué en dissolvant dans un récipient stérile $1 \mathrm{~g}$ de poudre dans $9 \mathrm{ml}$ d'eau distillée stérile portée préalablement à $45^{\circ} \mathrm{C}$. Après dissolution complète de la poudre, le lait était laissé au repos pendant $1 \mathrm{~h}$ à $25^{\circ} \mathrm{C}$ avant toute utilisation. La flore totale (dénombrée après incubation sur gélose nutritive $72 \mathrm{~h}$ à $30^{\circ} \mathrm{C}$ ) restait inférieure à 210 bactéries $/ \mathrm{ml}$ de lait et la flore psychrotrophe $\mathrm{C}$ dénombrée après incubation sur gélose nutritive $10 \mathrm{j}$ à $7^{\circ} \mathrm{C}$ ) ne dépassait pas 50 bactéries/ml de lait. Dans certains cas l'azoture de sodium, à une concentration finale de $0,2 \mathrm{~g} / \mathrm{l}$, était utilisé comme agent bactériostatique.

\section{Mesure de la stabilité du lait à l'alcool}

"L'épreuve à l'alcool " était réalisée de la façon suivante : à $2 \mathrm{ml}$ de lait reconstitué on ajoutait $2 \mathrm{ml}$ d'alcool éthylique à différents degrés Gay-Lussac. Rappelons que la concentration en degrés GayLussac est très peu différente de la concentration en p. 100 (v/v). Les concentrations utilisées étaient de $45,50,55,60,65,70,75,80$, 85,90 et $95^{\circ}$ G.L. Afin d'éliminer toute confusion d'interprétation, un mélange d'égal volume de lait et d'alcool à $\mathrm{x}^{\circ}$ G.L. sera désigné comme " épreuve à l'alcool à $x^{\circ}$ G.L. » (et jamais « épreuve à l'alcool à $\mathrm{x} / 2^{\circ} \mathrm{G}$.L. "). Les laits étaient considérés comme instables à l'alcool si des flocons apparaissaient en moins de $1 \mathrm{mn}$.

\section{Mesure de la stabilité du lait à la chaleur}

$55 \mu 1$ de lait étaient introduits par capillarité dans un tube de verre (diamètre intérieur $1 \mathrm{~mm}$, diamètre extérieur 1,2 $\mathrm{mm}$ ) (Kimax, réf. 34502 ) de $10 \mathrm{~cm}$ de longueur et de $78 \mu \mathrm{l}$ de contenance, puis les deux extrémités du tube étaient soudées à la flamme. Pour chaque mesure de la stabilité du lait à une température donnée, on préparait ainsi 10 tubes capillaires. Ces tubes étaient plongés dans un bain d'éthylène glycol régulé à $\pm 0,1^{\circ} \mathrm{C}$ (Ultrathermostat Haake). Au temps supposé de déstabilisation on observait toutes les $10 \mathrm{~s}$ s'il y avait ou non apparition de deux phases. Cette méthode permet d'estimer le temps de déstabilisation à \pm 5 p. 100. Les temps mesurés étant 
supérieurs à $1 \mathrm{mn}$, il n'a pas été nécessaire de tenir compte de l'effet de la période d'équilibrage des températures dans les tubes capillaires, qui est de l'ordre de $10 \mathrm{~s}$ (Scharer, 1965).

\section{Techniques de modification du lait}

\section{Acidification ou alcalinisation}

Les acidifications ont été effectuées en ajoutant sous agitation dans $1000 \mathrm{ml}$ de lait reconstitué des quantités croissantes d'acide chlorhydrique N/10 ou d'acide lactique N/10. Les alcalinisations ont été réalisées en ajoutant dans les mêmes conditions des quantités croissantes de soude $\mathrm{N} / 10$. En vue de recueillir une quantité représentative de la fraction soluble du lait après acidification ou alcalinisation sans modifier les équilibres salins, $1000 \mathrm{ml}$ étaient placés dans une cellule d'ultrafiltration équipée d'une membrane Diaflo UM-10 (Amicon). On récoltait $15 \mathrm{ml}$ d'ultrafiltrat sur lesquels le dosage des éléments minéraux était effectué.

\section{Ensemencement par des cellules microbiennes}

\subsection{Origine et conservation des souches}

Les souches utilisées dans ce travail provenaient de la collection de l'I.N.R.A. (C.N.R.Z.). Il s'agissait de : Streptococcus diacetylactis C.N.R.Z. 267, bactérie acidifiante à activité protéolytique faible (Desmazeaud et Zevaco, 1977), Micrococcus caseolyticus C.N.R.Z. 467, bactérie protéolytique acidifiant très lentement le lait (Desmazeaud et Hermier, 1968), Pseudomonas souche 37 (groupe putida fluorescens), bactérie protéolytique psychrotrophe alcalinisante. $S$. diacetylactis et $M$. caseolyticus étaient entretenus par repiquage sur lait écrémé tournesolé stérile et conservés par congélation à $-20^{\circ} \mathrm{C}$. Le Pseudomonas était conservé lyophilisé.

\subsection{Préparation des cellules et ensemencement du lait}

Le milieu utilisé pour la croissance de $S$. diacetylactis a été décrit précédemment par Bergère (1968). Pour $M$. caseolyticus on utilisait le milieu TG de Desmazeaud et Hermier (1968). Le Pseudomonas a été cultivé sur le milieu suivant : extrait de levure (Merck) $5 \mathrm{~g}$; glucose 5,5 g ; Bacto-tryptone (Difco) $10 \mathrm{~g}$; $\mathrm{K}_{2} \mathrm{HPO}_{4} 2 \mathrm{~g}$; eau distillée $1000 \mathrm{ml}$ (R. Mourgues, communication personnelle). Le $\mathrm{pH}$ du milieu était ajusté à 7,2 . Il était stérilisé à $120^{\circ} \mathrm{C}$ pendant $20 \mathrm{mn}$. Pour ces différentes espèces, trois repiquages successifs ont été effectués sur les milieux avant récolte des cellules. Celles-ci étaient récoltées en fin de croissance logarithmique par centrifugation à $8000 \mathrm{~g}$ pendant $15 \mathrm{mn}$. Elles étaient directement remises en suspension dans le lait reconstitué.

$S$. diacetylactis et $M$. caseolyticus ont été inoculés à raison de $5 \times 10^{5}$ et $4 \times 10^{5}$ cellules $/ \mathrm{ml}$ respectivement, puis incubés à $25^{\circ} \mathrm{C}$. Le Pseudomonas était ensemencé à raison de $1,3.10^{7}$ cellules $/ \mathrm{ml}$ et incubé à $7^{\circ} \mathrm{C}$. Ces cultures sur lait n'étaient pas agitées. 
Le nombre des cellules viables dans le lait ainsi ensemencé, puis au cours du temps, était déterminé par dénombrement sur Plate Count Agar (Difco) après $48 \mathrm{~h}$ à $30^{\circ} \mathrm{C}$ pour $S$. diacetylactis et $M$. caseolyticus et après $10 \mathrm{j}$ à $7^{\circ} \mathrm{C}$ pour le Pseudomonas.

\section{Dosages effectués et étude électrophorétique du lait}

1. Dosage de l'acide citrique

Il a été effectué selon la méthode de Marier et Boulet (1958) en employant le mélange pyridine-anhydride acétique. Après $30 \mathrm{mn}$ de réaction on mesurait la variation d'absorbance à $420 \mathrm{~nm}$.

\section{Dosage des chlorures}

Les chlorures étaient dosés dans l'ultrafiltrat défini ci-dessus, par la méthode de Charpentier-Vohlard (Serres et al., 1973).

3. Dosage du phosphore, du sodium, du calcium et du magnésium

Préalablement aux différents dosages, $10 \mathrm{ml}$ d'ultrafiltrat $(\S \mathrm{IV}, 1)$ étaient minéralisés à $530-550^{\circ} \mathrm{C}$ pendant $8 \mathrm{~h}$. Les résidus de la calcination étaient dissous dans $10 \mathrm{ml}$ d'acide nitrique à 10 p. 100 (v/v), puis portés à l'ébullition pendant $10 \mathrm{mn}$ sur bain de sable, sans aller à sec. Cette solution était filtrée sur papier sans cendres et le volume ajusté à $100 \mathrm{ml}$ avec de l'eau distillée (solution S1).

Le dosage du phosphore était effectué en mélangeant $10 \mathrm{ml}$ du réactif nitrovanadomolybdique à $10 \mathrm{ml}$ de la solution $\mathrm{S} 1$. Après $10 \mathrm{mn}$ de contact, l'intensité de la coloration était mesurée à $430 \mathrm{~nm}$. Le réactif nitrovanadomolybdique avait la composition suivante : molybdate d'ammonium à $100 \mathrm{~g} / 1: 200 \mathrm{ml}$; solution de métavanadate d'ammonium $5,85 \mathrm{~g} / 1$ : $200 \mathrm{ml}$; acide nitrique $(\mathrm{d}=1,38): 134 \mathrm{ml}$; eau distillée q.s.p. : $1000 \mathrm{ml}$.

A partir de la solution S1 le sodium était dosé par spectrophotométrie de flamme type air-propane, sur un appareil Eppendorf. Le calcium était dosé par spectrophotométrie de flamme type air-acétylène, sur un appareil Eppendorf. Le magnésium était dosé par absorption atomique avec une flamme type air-acétylène sur un appareil Instrumentation Laboratory 151 (L. Gueguen, communication personnelle).

\section{Dosage du calcium soluble ionisé}

A partir de l'ultrafiltrat défini ci-dessus $(\S I V, 1)$ le calcium ionisé était dosé selon la méthode de Seekles et Smeets (1947) en employant l'acide mercurique (murexide). Le changement de coloration était mesuré à $480 \mathrm{~nm}$.

\section{Dosage des groupes $\alpha$-aminés libres}

Préalablement aux dosages, les protéines et les peptides de haut poids moléculaire étaient précipités par l'acide trichloracétique à une 
concentration finale de 12 p. $100(\mathrm{~m} / \mathrm{v})$. Après $1 \mathrm{~h}$ de contact la suspension était filtrée sur papier Whatman 42. Les groupes $\alpha$-aminés libres ont été dosés, sur le filtrat dilué 5 fois, par le réactif à la ninhydrine selon la méthode de Moore et Stein (1954). La mesure de l'absorbance de la coloration apparue se faisait à $570 \mathrm{~nm}$.

\section{Etude électrophorétique du lait}

Les électrophorèses étaient réalisées sur le lait lui-même en gel mixte d'acrylamide-agarose selon la technique d'Uriel (1966) modifiée par l'utilisation d'urée (Gripon et al., 1975). La coloration avec le bleu de Coomassie et le lavage consécutif des gels ont été effectués selon la méthode d'Uriel (1966).

\section{Présentation des résultats des mesures de stabilité}

Pour caractériser la stabilité à la chaleur, c'est le temps pour obtenir la déstabilisation à une température donnée qui est présenté. Pour caractériser la stabilité à l'alcool, on a indiqué la concentration en alcool (exprimée en degrés Gay-Lussac) de la solution mélangée au lait qui provoque la déstabilisation de ce dernier.

\section{RESULTATS}

\section{Caractéristiques du lait sec écrémé utilisé}

Le lait utilisé dans ce travail avait les caractéristiques suivantes : $\mathrm{pH} 6,66$; acidité titrable $16,5^{\circ}$ Dornic. Il était instable à l'épreuve à l'alcool à $80^{\circ}$ G.L. mais stable à $75^{\circ}$ G.L.

La stabilité à la chaleur a été recherchée sur des laits contenant 0,02 p. 100 d'azoture de sodium, de $129,7^{\circ} \mathrm{C}$ à $156^{\circ} \mathrm{C}$. Il a été vérifié que l'azoture de sodium n'avait pas d'influence sur le phénomène. Comme il apparaît sur la figure 1 (représentation d'Arrhenius) la stabilité à la chaleur dépend d'au moins deux phénomènes, le premier se manifestant en dessous de $150^{\circ} \mathrm{C}$ et le deuxième au-dessus de $150^{\circ} \mathrm{C}$. L'énergie d'activation du deuxième phénomène $\left(\mathrm{E}^{*}=252 \mathrm{~kJ} /\right.$ mol $\left.; \mathrm{z}=13,7^{\circ} \mathrm{C}\right)$ est plus élevée que celle du premier $\left(\mathrm{E}^{*}=119 \mathrm{~kJ} /\right.$ $\left.\mathrm{mol} ; \mathrm{z}=27,4^{\circ} \mathrm{C}\right)$. Ainsi, la température de $140^{\circ} \mathrm{C}$ pour laquelle le temps de déstabilisation est de $26 \mathrm{mn}$, s'annonce comme étant la mieux appropriée pour effectuer les tests de stabilité à la chaleur dans le cadre de ce travail. En effet, cette température est fréquemment utilisée pour les traitements U.H.T. industriels et surtout offre une précision suffisante pour la mesure du temps de déstabilisation des laits qui ont été le siège d'une croissance microbienne. 


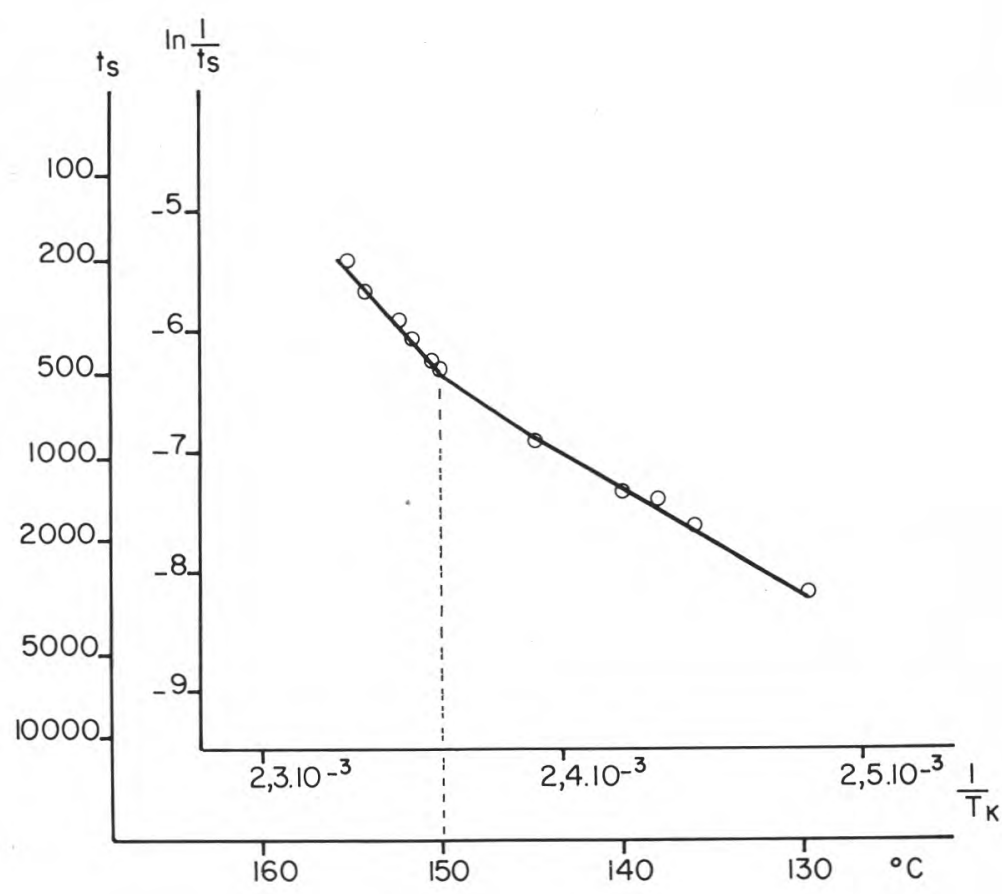

fig. 1

Temps de déstabilisation du lait reconstitué en fonction de la température

En abscisses: inverse des températures en Kelvin (avec correspondance en degrés Celsius).

En ordonnées : logarithme népérien de l'inverse des temps de déstabilisation en secondes (avec correspondance des temps en secondes). Les énergies d'activation d'Arrhenius, notées $E^{*}$, de la déstabilisation $\mathrm{du}$ lait sont proportionnelles aux pentes de la courbe :

de $129,7^{\circ} \mathrm{C}$ à $150^{\circ} \mathrm{C}, \mathrm{E}^{*}=119 \mathrm{~kJ} / \mathrm{mol}$. de $150^{\circ} \mathrm{C}$ à $156^{\circ} \mathrm{C}, \mathrm{E}^{*}=252 \mathrm{~kJ} / \mathrm{mol}$.

\section{Influence de l'acidification ou de l'alcalinisation}

Après une acidification de $\mathrm{pH} 6,66$ à $\mathrm{pH} 6,18$ on observait une baisse progressive de la stabilité à l'alcool de $80^{\circ}$ G.L. à $55^{\circ}$ G.L. alors que la baisse de la stabilité à $140^{\circ} \mathrm{C}$ s'effectuait en deux phases. En effet de pH 6,66 à pH 6,4 la décroissance était assez faible alors qu'elle était brutale en dessous de $\mathrm{pH} \mathrm{6,40} \mathrm{(fig.} \mathrm{2).}$

Après une alcalinisation de $\mathrm{pH} 6,66$ à $\mathrm{pH} 7,14$ on observait une augmentation progressive de la stabilité à l'alcool jusqu'à $95^{\circ}$ G.L. 

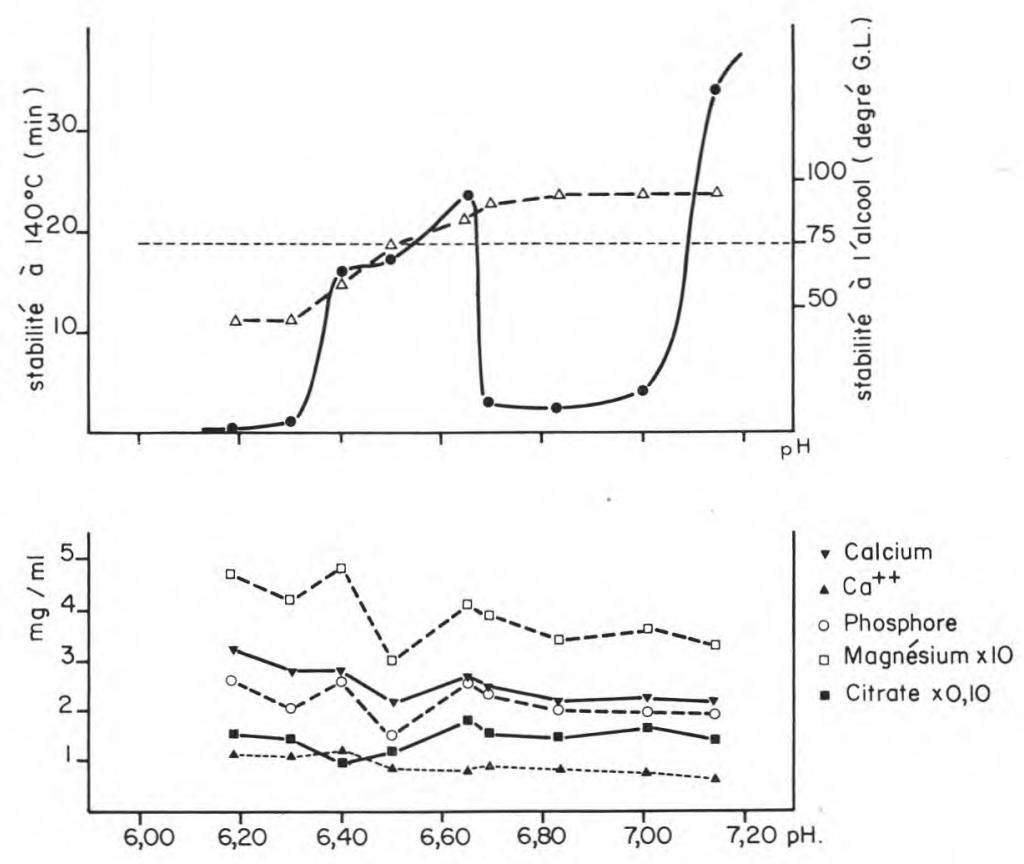

fig. 2

Effet de l'acidification et de l'alcalinisation du lait sur sa stabilité à l'alcool et sa stabilité à $140^{\circ} \mathrm{C}$ ainsi que sur les concentrations en certains éléments minéraux de la fraction soluble.

$\triangle$ (degrés G.L. de l'alcool ajouté provoquant la déstabilisation).

- (temps de déstabilisation en minutes à $140^{\circ} \mathrm{C}$ ).

Par contre, la stabilité à la chaleur décroissait fortement de $\mathrm{pH}$ 6,66 à $\mathrm{pH}$ 6,69, puis croissait brutalement à partir de $\mathrm{pH}$ 7,01 (fig. 2). Par ailleurs on a montré qu'une acidification artificielle par de l'acide lactique entraînait les mêmes variations de stabilité que celles obtenues par de l'acide chlorhydrique. Que ce soit par acidification ou par alcalinisation on n'observait pas de variation nette des concentrations en éléments minéraux solubles du lait (fig. 2).

\section{Influence de la croissance de bactéries lactiques mésophiles}

Le développement de $S$. diacetylactis 267 entraînait une baisse progressive du $\mathrm{pH}$ et de la stabilité à l'alcool de $80^{\circ}$ G.L. à $50^{\circ}$ G.L. (fig. 3). Au cours de cette acidification la stabilité à la chaleur restait bonne (de l'ordre de $17 \mathrm{mn}$ à $140^{\circ} \mathrm{C}$ ) jusqu'à $\mathrm{pH} 6,43$. Par contre, 

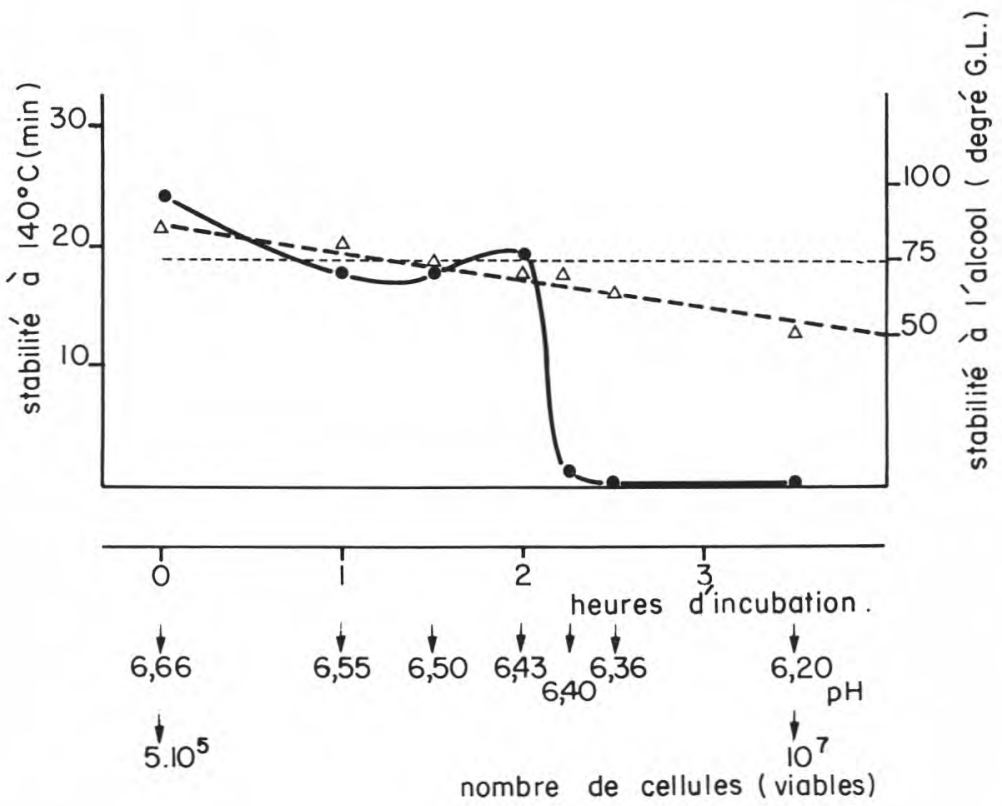

fig. 3

Influence de la croissance de Streptococcus diacetylactis 267 à $25^{\circ} \mathrm{C}$ dans le lait sur sa stabilité à la chaleur et sa stabilité à l'alcool.

$\triangle$ (degrés G.L. de l'alcool ajouté provoquant la déstabilisation).

- (temps de déstabilisation en minutes à $140^{\circ} \mathrm{C}$ ).

On notera que sur cette figure et les suivantes, les échelles en abscisses sont disposées dans le sens correspondant à l'augmentation du nombre de cellules microbiennes. De ce fait, l'acidification augmente de la gauche vers la droite, au contraire de ce qui est présenté sur la figure 2.

à partir de cette valeur elle chutait brutalement, le lait se déstabilisant quasi instantanément à $140^{\circ} \mathrm{C}$, à partir de $\mathrm{pH}$ 6,36 (fig. 3). Donc l'action de $S$. diacetylactis 267 était tout à fait comparable à celle observée dans le cas d'une acidification artificielle (fig. 2). En particulier, bien qu'encore stable pendant $17 \mathrm{mn}$ à $140^{\circ} \mathrm{C}$, le lait devient instable à l'épreuve à l'alcool à $75^{\circ}$ G.L. dès que le $\mathrm{pH}$ atteint 6,50 (fig. 3). Par ailleurs on n'observait aucune protéolyse puisqu'il n'y avait ni variation des électrophorégrammes du lait ni apparition de groupes $\alpha$-aminés au cours de la croissance de $S$. diacetylactis 267. 


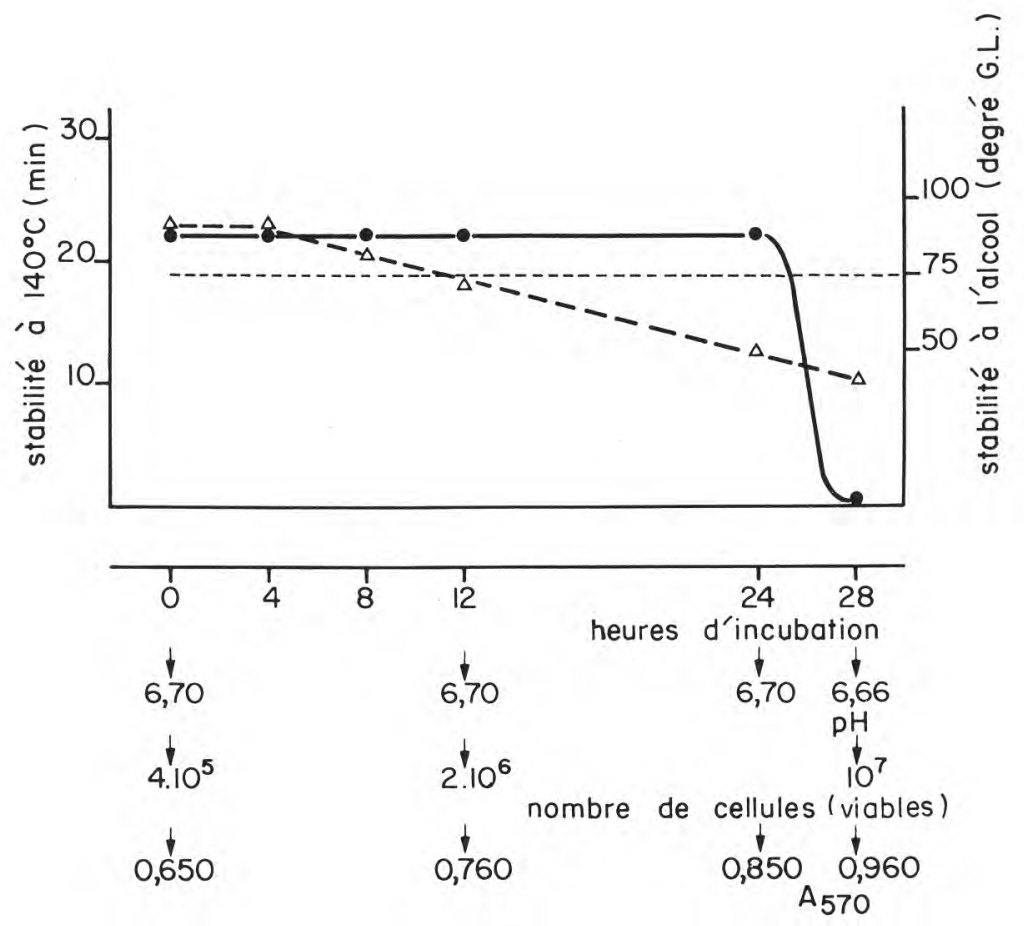

fig. 4

Influence de la croissance de Micrococcus caseolyticus 467 à $25^{\circ} \mathrm{C}$ dans le lait sur sa stabilité à la chaleur, sa stabilité à l'alcool et sur sa teneur en résidus aminés libres.

$\triangle$ (degrés G.L. de l'alcool ajouté provoquant la déstabilisation).

- (temps de déstabilisation en minutes à $140^{\circ} \mathrm{C}$ ).

$\mathrm{A}_{570}$ (absorbance à $570 \mathrm{~nm}$ indiquant la variation de la teneur en résidus aminés libres).

\section{Influence de la croissance de microcoques protéolytiques}

La présence et le développement de $M$. caseolyticus 467 entrâ̂naient une baisse progressive de la stabilité du lait à l'alcool allant de $80^{\circ}$ G.L. à $45^{\circ}$ G.L. En particulier le lait devenait instable à l'épreuve à l'alcool à $75^{\circ}$ G.L. lorsqu'on dénombrait $10^{6}$ cellules $/ \mathrm{ml}$ environ. Par contre, le lait ne devenait instable à la chaleur qu'après 24 h d'incubation. Au cours de cette période, aucune variation de $\mathrm{pH}$ n'était enregistrée mais on notait l'apparition progressive de groupes $\alpha$-aminés libres (fig. 4) dosés par le réactif à la ninhydrine dès $4 \mathrm{~h}$ d'incubation. Ce résultat traduit la production dans le lait d'une enzyme protéolytique dont l'action sur les caséines a été recherchée par électropho- 


\section{fig. 5}

Electrophorégramme des protéines du lait modifiées par la croissance de Micrococcus caseolyticus M96 pendant $24 \mathrm{~h}$ à $25^{\circ} \mathrm{C}$.

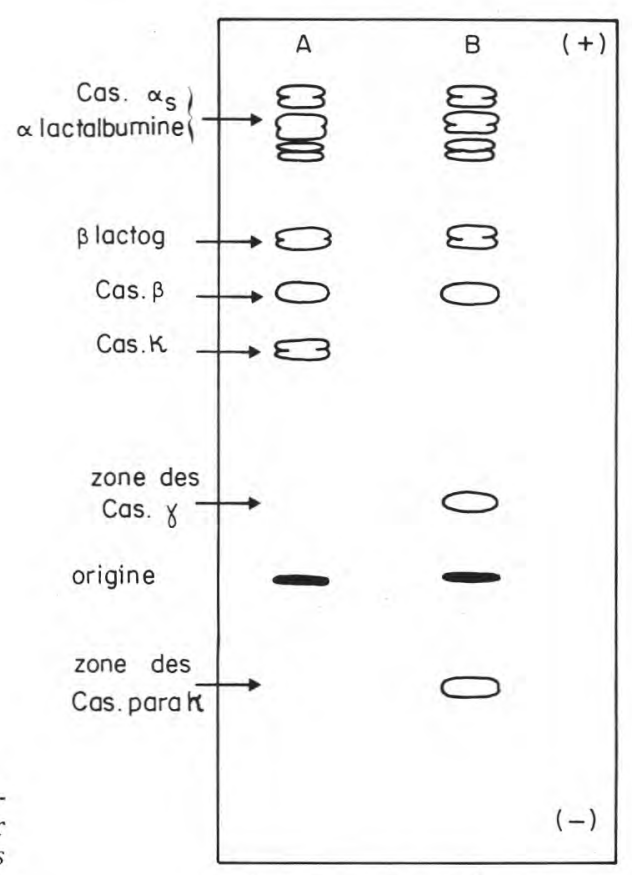

A: Témoin B: après $24 \mathrm{~h}$ de culture

rèse. En effet après $24 \mathrm{~h}$ de croissance à $25^{\circ} \mathrm{C} M$. caseolyticus entraînait une hydrolyse totale de la caséine $\mathrm{K}$ et la formation de composés apparaissant dans la zone des caséines para-K ou celle des caséines $\gamma$ (fig. 5). Par contre, aucune attaque des autres protéines du lait n'était mise en évidence au cours de cette période.

\section{Influence de la croissance de Pseudomonas psychrotrophes et protéolytiques}

Une augmentation du nombre des cellules viables de Pseudomonas 37 n'entraînait que très tardivement une instabilité du lait à l'alcool. En effet, elle commençait à partir de $10^{9}$ cellules $/ \mathrm{ml}$ et il fallait atteindre $10^{10}$ cellules/ml pour que le lait soit déstabilisé à l'épreuve à l'alcool à $65^{\circ}$ G.L. (fig. 6). D'autre part, la présence de cette bactérie psychrotrophe entraînait une augmentation immédiate et progressive du $\mathrm{pH}$ du lait de $\mathrm{pH} 6,70$ à $\mathrm{pH}$ 6,82 en 8 j à $7^{\circ} \mathrm{C}$. Simultanément; on observait une baisse immédiate et continue de la stabilité du lait à la chaleur puisqu'elle passait de $25 \mathrm{mn}$ à $7 \mathrm{mn}$ (à $140^{\circ} \mathrm{C}$ ) en $2 \mathrm{j}$ de culture à $7^{\circ} \mathrm{C}$ ou à $3 \mathrm{mn}$ après $5 \mathrm{j}$ d'incubation à $7^{\circ} \mathrm{C}$. Par ailleurs, 

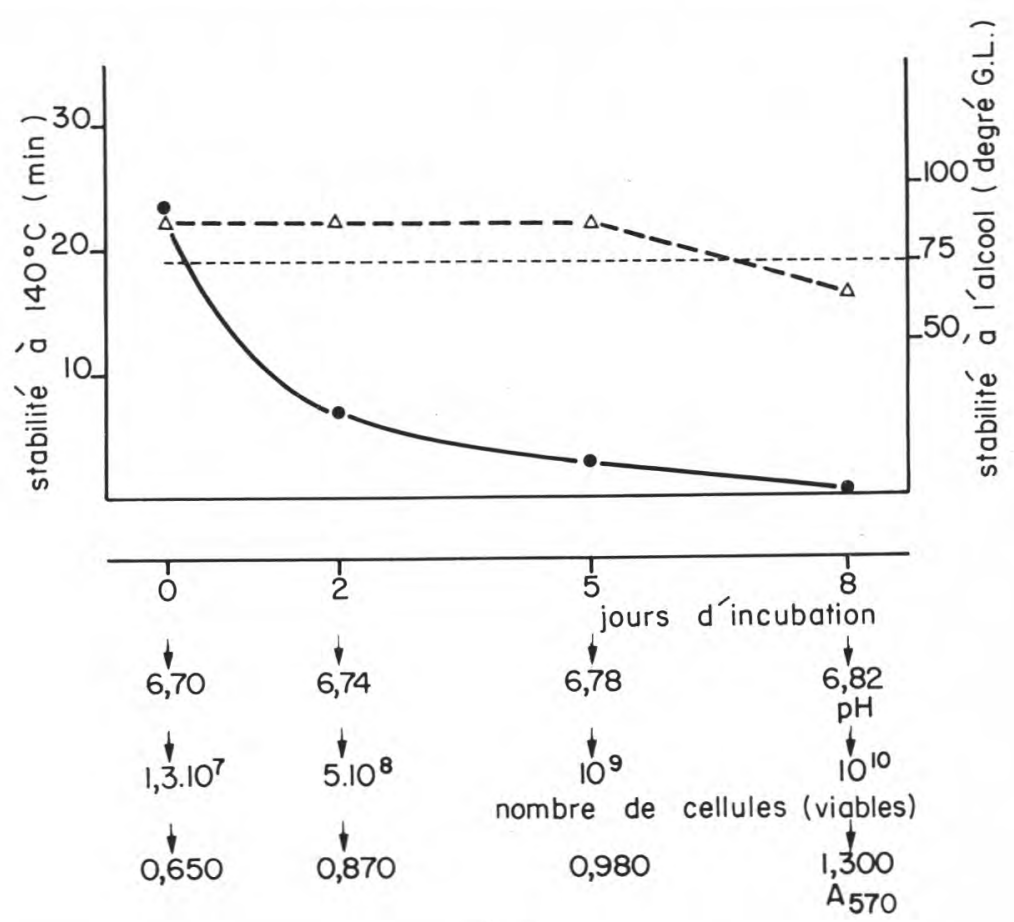

fig. 6

Influence de la croissance de Pseudomonas 37 à $7^{\circ} \mathrm{C}$ dans le lait sur sa stabilité à la chaleur, sa stabilité à l'alcool et sur sa teneur en résidus aminés libres.

$\triangle$ (degrés G.L. de l'alcool ajouté provoquant la déstabilisation).

- (temps de déstabilisation en minutes à $140^{\circ} \mathrm{C}$ ).

$\mathrm{A}_{570}$ (absorbance à $570 \mathrm{~nm}$, indiquant la variation de la teneur en résidus aminés libres).

on observait corrélativement une forte augmentation du nombre des groupes $\alpha$-aminés libres dans le lait ce qui traduit l'action d'enzyme(s) protéolytique(s) (fig. 6). Comme dans le cas de la présence de microcoques, l'action de cette protéase a été recherchée par électrophorèse du lait.

Comme il est montré figure 7, la croissance de Pseudomonas 37 entraînait dans le lait une hydrolyse importante des caséines. En particulier, dès le $2^{\mathrm{e}}$ jour la caséine $\mathrm{K}$ commençait à être hydrolysée. Après $5 \mathrm{j}$ à $7^{\circ} \mathrm{C}$, une forte hydrolyse de cette caséine était observée et l'on notait aussi une hydrolyse de la caséine $\alpha_{\text {si }}$ ainsi que l'apparition de plusieurs composés dans la zone de migration des caséines $\gamma$ 


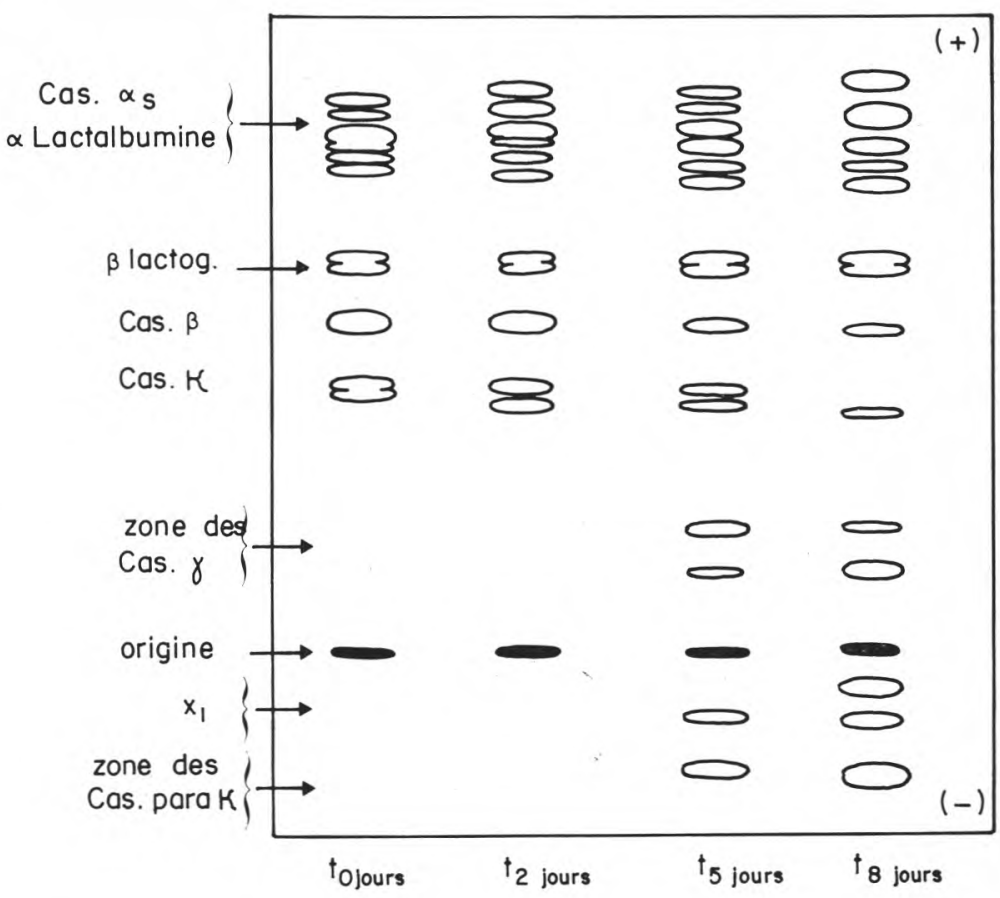

fig. 7

Electrophorégramme des protéines du lait modifiées par la croissance de Pseudomonas 37, montrant une hydrolyse progressive des caséines $\alpha_{s}$, $\beta$, et $\mathrm{K}$ au cours d'une incubation de $8 \mathrm{j}$ à $7^{\circ} \mathrm{C}$.

et para-K. Après $8 \mathrm{j}$ d'incubation du lait à $7^{\circ} \mathrm{C}$ cette hydrolyse s'était accentuée et l'on notait la quasi-disparition des caséines $K$ et $\beta$ et l'apparition d'un nouveau composé cationique.

\section{DISCUSSION}

Bien qu'il ait subi des traitements thermiques, le lait reconstitué choisi pour les essais a des caractéristiques représentatives de celles des laits crus individuels ou de grand mélange étudiés par d'autres auteurs (Fox et Morrissey, 1977). Ainsi le coefficient de température 
moyen pour l'ensemble de l'intervalle étudié est $\mathrm{Q}_{10}=3$. De même le temps de déstabilisation aux diverses températures comme la stabilité à l'alcool peuvent être considérés comme normaux (fig. 1 et 2). Son comportement à la chaleur à $140^{\circ} \mathrm{C}$ lorsque le $\mathrm{pH}$ varie (fig. 2) est celui des laits de type A dont la courbe des temps de coagulation par la chaleur est caractérisée par deux minima $(\mathrm{pH}<6,3 ; \mathrm{pH}=6,8)$ et deux maxima ( $\mathrm{pH} 6,4$ à 6,$6 ; \mathrm{pH}>7,1)$.

La figure 2 a fait ressortir par ailleurs qu'à certaines valeurs du $\mathrm{pH}(6,4$ ou 6,6 par exemple), une faible variation de ce dernier entraîne une forte variation de la stabilité à la chaleur. En revanche la variation de la stabilité à l'alcool est progressive. Ainsi, lorsque le $\mathrm{pH}$ descend de 6,40 à 6,30 , le temps de déstabilisation thermique passe de 17 à $2 \mathrm{mn}$, tandis que la stabilité à l'alcool varie de 60 à $45^{\circ}$ G.L. On remarque aussi qu'à $\mathrm{pH} 6,4$, le lait serait qualifié d'instable à l'alcool, alors que sa stabilité à la chaleur est encore excellente. C'est pour de telles raisons que Vaitkus et al. (1974) refusent d'utiliser l'épreuve à l'alcool comme critère de stabilité à la chaleur. D'autre part on ne retrouve pas les relations attendues entre la stabilité à la chaleur et le rapport Ca soluble/P soluble, ni la corrélation négative entre la stabilité à l'alcool et $\mathrm{Ca}$ ionisé. Ceci est une indication que les conclusions tirées des études de laits individuels ne peuvent être extrapolées simplement à un lait donné dont les caractéristiques sont modifiées.

Dans d'autres essaís (Métro, 1978), les stabilités à l'alcool et à la chaleur ont été mesurées après diverses modifications du lait : augmentation de la concentration en matière sèche par reconstitution avec davantage de poudre de lait ou par ultrafiltration, diminution de la concentration en matière sèche par reconstitution avec moins de poudre le lait ou par dialyse. Une conclusion analogue a pu être tirée de ces essais : aucune relation entre stabilité et composition n'a pu être trouvée.

Cependant les modifications du lait par concentration ou dilution sont peu probables dans les conditions technologiques habituelles. C'est donc sur les modifications qui sont provoquées par des microorganismes que nous insisterons. Les trois souches étudiées sont susceptibles de se développer dans le lait en tank de ferme, c'est-à-dire à des températures oscillant entre celle du lait à la traite et $4^{\circ} \mathrm{C}$, puis entre $4^{\circ} \mathrm{C}$ et la température dans le tank lorsqu'on lui rajoute du lait fraîchement trait. Pour rendre plus évidents les effets de la présence des bactéries, les nombres inoculés étaient beaucoup plus élevés que ceux qui se trouvent en général dans le lait notamment pour les bactéries psychrotrophes.

La comparaison entre la figure 2 d'une part et les figures 3 et 6 de l'autre a montré un parallélisme remarquable entre les modifications des stabilités à la chaleur et à l'alcool produites par l'acidification et l'alcalinisation, que celles-ci aient été obtenues par addition d'acide ou d'alcali, ou qu'elles aient été obtenues par croissance de 
S. diacetylactis ou de Pseudomonas 37. Lors de l'acidification, le lait reste longtemps stable à la chaleur. Avec la croissance de $M$. caseolyticus, la déstabilisation à la chaleur se produit à un $\mathrm{pH}$ plus élevé qu'avec $S$. diacetylactis, quoique après une incubation beaucoup plus longue aboutissant notamment à l'hydrolyse de la caséine.

I1 ressort des comparaisons qui précèdent que la sélection du lait au moyen de l'épreuve à l'alcool à $75^{\circ}$ G.L. a une efficacité très différente selon le type de flore microbienne qui domine. Avec une flore dont l'activité essentielle est de produire de l'acide, telle que la flore lactique qui se développe rapidement à température ambiante (comme cela se passe par exemple pendant la collecte classique en bidon), l'épreuve à l'alcool donne un résultat sûr. En effet le lait perd sa stabilité à l'alcool bien avant sa stabilité à la chaleur. L'écart entre les deux déstabilisations est encore plus grand dans le cas du développement de microcoques mésophiles protéolytiques.

Au contraire, avec une flore psychrotrophe protéolytique du type de celle étudiée (caractéristique des tanks de ferme), le lait perd sa stabilité à la chaleur très vite, longtemps avant la stabilité à l'alcool. Dans ce cas, celle-ci ne permet pas la sélection du lait. Cette dernière affirmation a été vérifiée dans une usine recevant exclusivement des laits réfrigérés. Toutefois d'autres confirmations devraient être faites avant de permettre une généralisation dans les conditions de la pratique. Il est d'ailleurs vraisemblable que les effets de flores mixtes soient additifs.

Les essais réalisés au cours de cette étude montrent par ailleurs que ni la mesure du $\mathrm{pH}$, ni celle de la protéolyse par dosage des résidus aminés libérés par exemple, ne peuvent servir d'indice de la stabilité à la chaleur. C'est pourquoi il nous semble prudent, dans les cas où l'épreuve à l'alcool donne des résultats peu fiables, de mesurer directement la stabilité à la chaleur, par exemple au moyen de la technique utilisée pour ce travail.

De façon à obtenir rapidement la réponse, une température supérieure à $140^{\circ} \mathrm{C}$ pourrait être choisie, $150^{\circ} \mathrm{C}$ constituant une valeur à ne pas dépasser (voir plus haut). Toutefois, il n'est pas possible de proposer a priori une valeur universelle limite pour le temps de déstabilisation; chaque usine devra étalonner la technique en fonction du type de traitement thermique industriel qu'elle applique.

\section{Remerciements}

Nous remercions vivement $M$. L. Guéguen qui nous a donné les moyens d'effectuer les dosages minéraux, Mme $Y$. Vassal et $M$. $R$. Mourgues pour la fourniture des souches ainsi que MM. J. Hermier et J. L. Bergère pour leur aide dans la préparation du manuscrit. 


\section{Ré s u mé}

La stabilité à l'alcool et à la chaleur d'un lait reconstitué ont été mesurées en fonction de la croissance de bactéries. Les souches suivantes ont été utilisées : (1) Streptococcus diacetylactis C.N.R.Z. 267, (2) Micrococcus caseolyticus C.N.R.Z. 467, cultivées à $25^{\circ} \mathrm{C}$ et (3) Pseudomonas (groupe putida fluorescens) souche 37 , cultivée à $7^{\circ} \mathrm{C}$. Avec (1), il y a acidification forte du lait et pas de protéolyse décelable : la stabilité à l'alcool est perdue un peu avant la stabilité à la chaleur. Avec (2), l'acidification est faible, et une protéolyse a lieu : la stabilité à l'alcool est perdue longtemps avant la stabilité à la chaleur. Avec (3), il y a une forte protéolyse et une légère alcalinisation : la stabilité à la chaleur est perdue très tôt, longtemps avant la stabilité à l'alcool. Ces résultats montrent que l'épreuve à l'alcool peut ne pas être un bon critère de stabilité à la chaleur, surtout lorsque la collecte du lait est réalisée dans des conditions qui ne sont pas favorables à la flore acidifiante mésophile. La vérification directe de la stabilité à la chaleur semble donc préférable.

\section{S u m m a r y}

\section{FACTORS INFLUENCING THE VALIDITY OF THE ALCOHOL TEST USED FOR SELECTING HEAT STABLE MILK}

Alcohol and heat stability of reconstituted milk was measured after bacterial growth. The following strains were used : (1) Streptococcus diacetylactis C.N.R.Z. 267, (2) Micrococcus caseolyticus C.N.R.Z. 467 , incubated at $25^{\circ} \mathrm{C}$, and (3) Pseudomonas (putida-fluorescens group) strain 37 incubated at $7^{\circ} \mathrm{C}$. With (1), there was a strong acidification and no detectable proteolysis: alcohol stability was lost shortly before heat stability. With (2), there was a slight acidification, and a proteolysis was noted: alcohol stability was lost long before heat stability. With (3), there was a strong proteolysis and a slight alcalinization : heat stability was lost very soon, long before alcohol stability. These results show that the alcohol test may be a bad criterium for heat stability, mainly when milk collecting conditions are not favourable to mesophilic acidifying bacteria. Direct testing of heat stability is recommended.

Reçu pour publication en mai 1979.

\section{Bibliographie}

BATRA (S. C.) and MAN (J. M. DE) (1964). - The interrelationship between rennet clotting time, alcohol stability and mineral constituents of milk. Milchwissenschaft, 19, 531-535. 
Belousov (A. P.), Surotsev (A. V.) et Rossikhina (G. A.) (1971). - Thermostabilité du lait et sa détermination (en russe). Molochnaya Promyshlennost, 32 (1), 6-9.

Bergère (J. L.) (1968). - Production massive de cellules de streptocoques lactiques. I. Méthodes générales d'étude et facteurs de croissance de Streptococcus lactis souche C 10. Lait, 48, 1-11.

Claesson (C. O.), Andersson (K.) et Claesson (E.) (1974). - Variations des propriétés du lait et leur influence sur le traitement U.H.T. XIX Congrès international de Laiterie, $1 \mathrm{~F}, 213$.

Davies (D. T.) and White (J. C. D.) (1958). - The relation between the chemical composition of milk and the stability of the caseinate complex. II. Coagulation by ethanol. Journal of Dairy Research, 25, 256-266.

Desmazeaud (M.) et Hermier (J.) (1968). - Facteurs intervenant dans la production du système protéolytique chez Micrococcus caseolyticus. Annales de Biologie animale, Biochimie, Biophysique, 8, 419-429.

Desmazeaud (M. J.) and Zevaco (C.) (1977), - General properties and substrate specificity of an intracellular soluble dipeptidase from Streptococcus diacetilactis. Annales de Biologie animale, Biochimie, Biophysique, 17, 723-726.

FoX (P. F.) and MORRIssey (P.A.) (1977). - Review of the progress of dairy science: The heat stability of milk. Journal of Dairy Research, 44, 627-646.

Gripon (J. C.), Desmazeaud (M. J.), Le Bars (D.) et Bergère (J. L.) (1975). Etude du rôle des micro-organismes et des enzymes au cours de la maturation des fromages. II. Influence de la présure commerciale. Lait, 55, 502-516.

Habaj (B.), Fetlinski (A.), Damicz (W.) et Dziuba (J.) (1974). - Facteurs d'instabilité des protéines du lait cru dans le test à l'alizarine. XIX $X^{\mathrm{e}}$ Congrès international de Laiterie, 1 F, 207-208.

Iızuka (M.) (1960), - Studies of alcohol test positive milk with normal acidity. I. Field survey on alcohol tested positive milk of dairy cows. Japanese Journal of veterinary Science, 22, 369-380. In Dairy Science Abstracts, 23, 2067.

Jooste (P. J.) and Groeneveld (H. T.) (1971). - Bacterial counts and dye reduction tests in relation to the keeping quality of South African pasteurized milk. South African Journal of Dairy Technology, 3, 193-201. In Dairy Science Abstracts, 34, 3779.

Kisza (J.), KRUK (A.), und Roskosz (A.) (1974). - Untersuchungen über die Hitzestabilität normaler und Mastitismilch. Milchwissenschaft, 29, 141-145.

Labuschagne (J. H.) (1968). - The stability of the caseinate complex in milk. I. The relationship between alcohol stability and the mineral composition (en affrikaans). Dairy Industry Journal of Africa, 8, 155-157, 159-161 and 163. In Dairy Science Abstracts, 32, 2230 ,

Labuschagne (J. H. A.) and Landrey (J.S. A.) (1969). - The stability of the caseinate complex in milk. II. Influence of certain feeding treatments on the stability of milk. South African Journal of Dairy Technology, 1, 31-37. In Dairy Science Abstracts, 32, 2231.

MARIER (J.R.) and Boulet (M.) (1958). - Direct determination of citric acid in milk with an improved pyridine-acetic anhydride method. Journal of Dairy Science, 41, 1683-1692.

Métro (F.) (1978). - Corrélation entre la stabilité à l'alcool et la stabilité à la chaleur du lait. Mémoire d'Ingénieur C.N.A.M., 117 p.

MoORE (S.) and STEIN (W. H.) (1954), - A modified ninhydrin reagent for the photometric determination of amino-acids and related compounds. Journal of biological Chemistry, 211, 907-913.

Puri (B. R.), Muraki (K.) and Toteja (K. K.) (1965). - Studies in stability of protein dispersion in milk. I. Ethanol stability. Indian Journal of Dairy Science, 18, 112-114. In Dairy Science Abstracts, 28, 2939. 
Seekles (L.) and Smeets (W. Th. G. M.) (1947). - Instability of milk due to an increased activity of calcium ions. Netherlands' Milk and Dairy Journal, $1,7-8$.

Serres (L.), Amariglio (S.) et Petransxiene (D.) (1973). - Contrôle de la qualité des produits laitiers. Tomes I et II. Paris : Informations techniques des Directions des Services vétérinaires.

SchARER (J. M.) (1965). - Thermal death behavior of bacterial spores. Thesis, University of Pennsylvania.

Toit (J. J. DU), Lück (H.) and Tonder (J. L. Van) (1970). - The relationship between the bacterial content of milk and its alcohol stability. South African Journal of Dairy Technology, 2, 193-199. In Dairy Science Abstracts, 33, 990.

URIEL (J.) (1966). - Méthode d'électrophorèse dans des gels d'acrylamide-agarose. Bulletin de la Société de Chimie biologique, 48, 969-982.

Vaitkus (V.), Kaplanas (R.), Kaplanene (B.), Yarmolenko (V.) (1974). - Study of heat stability of milk proteins. I. Dependance on $\mathrm{pH}$ and milk fat dispersion (en russe). Trudy Litovskii Filial Vsesoyuznogo Nauchno issledovatel'skogo Instituta Maslodel'noi i Syrodel'noi Promyshlennosti, 9, 87-93, 227. In Dairy Science Abstracts, 37, 5092.

Zhdanova (E. A.) and Alekseeva (N. Yu.) (1969). - Correlation between the stability of milk in the alcohol test and some other milk characteristics (en russe). Molochnaya Promyshlennost, 30 (5), 23-26. 